\title{
Identification and physical mapping of induced translocation breakpoints involving chromosome $1 R$ in rye
}

\author{
S. Catarino ${ }^{1}$, E. Alvarez ${ }^{2}$, A. Campa ${ }^{2}$, R. Vieira ${ }^{1}$, A. Roca $^{2}$ \& R. Giraldez ${ }^{2 *}$ \\ ${ }^{1}$ Instituto Botânico, Universidade de Coimbra, 3000-000 Coimbra, Portugal; ${ }^{2}$ Departamento de Biología \\ Funcional, Universidad de Oviedo, 33006 Oviedo, Spain; Tel: +34-985103594; Fax: +34-985103534; \\ E-mail: giraldez@uniovi.es \\ S. Catarino and E. Alvarez contributed equally to this work \\ * Correspondence
}

Received 6 July 2006. Received in revised form and accepted for publication by Steve Stack 8 September 2006

Key words: physical mapping, rye, synaptonemal complex, translocation breakpoint

\begin{abstract}
To obtain translocations involving specific chromosomes in rye, pollen of a line in which chromosome $1 \mathrm{R}$ has large C-bands on its two telomeres, but which lacks C-bands (or has very small ones) on the telomeres of the remaining chromosomes, was X-irradiated. All translocations involving the labelled chromosome (1R) could be easily recognized in C-banded mitotic metaphases. The non-labelled chromosome involved in each translocation was identified either from mitotic C-banding analysis or from the meiotic configurations observed in some specific progenies. A physical map including 40 translocation breakpoints has been developed by means of synaptonemal complex (SC) analysis of well-paired pachytene quadrivalents. The results agree with the hypothesis of chromosomes $2 \mathrm{R}$ to $7 \mathrm{R}$ having similar probabilities of participating in translocations with chromosome 1R. However, the locations of the breakpoints are not entirely random: an excess of translocation breakpoints located on the short arm of chromosome $1 \mathrm{R}$ was obtained, and the two acentric translocated segments of each translocation show a trend towards having similar sizes. The possible reasons for these two non-random situations are discussed.
\end{abstract}

\section{Introduction}

Chromosome rearrangements, like translocations or deletions, are useful tools for the development of physical maps in plants. Since only polyploid plants can tolerate the majority of the deletions, translocations have been the mutations most extensively used in cytogenetic mapping of diploid plants (de Vries \& Sybenga 1984, Linde-Laursen 1988, Hoisington \& Coe 1990, Sybenga et al. 1990, Alonso-Blanco et al. 1993a,b, 1994, Sorokin et al. 1994, Künzel et al. 2000).

A translocation set covering all seven rye chromosomes was constructed by Sybenga \& Wolters (1972). This set was extended by Ramulu \& Sybenga
(1985), and a few translocations of spontaneous origin were later described by Alvarez et al. (1994). However, the collection of translocations in rye is much smaller than in barley (Linde-Laursen 1988, Künzel 1992) or maize (Longley 1961) in which a high degree of saturation for all chromosomes has been reached.

Rye translocations may be efficiently induced by pollen irradiation with X-rays (Sybenga \& Wolters 1972) but their later identification may represent a laborious and difficult task. Since most rye chromosomes show small differences in their C-band patterns, only reciprocal exchanges giving rise to size differences in the translocated chromosomes can be unequivocally 
identified at mitosis. The presence of quadrivalents in meiosis can also be used to identify plants carrying translocated chromosomes, but this method requires the cultivation of a large number of plants until blooming. In some plant species, such as maize and barley, the selection of translocation heterozygotes has been based on their semisterility resulting from meiotic irregularities. However, this criterion is not of use in rye, because translocations heterozygotes in this species tend to be rather fertile as a consequence of the relatively frequent alternate orientation of the quadrivalent at meiosis (Sybenga 1995).

Alvarez et al. (1997) proposed a method for the induction and identification of rearrangements involving a specific chromosome in rye. This method was based on pollen $\mathrm{X}$-irradiation of plants belonging to a line having large telomere C-bands only in chromosome 1R (line 1RSL). With this strategy all translocations involving the labelled chromosome could be easily recognized in C-banded mitotic metaphases.

In this work, 20 new translocations involving chromosome 1R, obtained through the above-indicated strategy, have been characterized. The identification of the non-labelled chromosomes involved in each translocation was made by the analysis of either the Cbanding pattern of mitotic chromosomes, or meiotic metaphase I configurations of some specific progenies. The relative position of the breakpoints within the chromosomes involved in each translocation was determined from the analysis of pachytene synaptonemal complexes (SC) of the quadrivalents formed in translocation heterozygotes. The final goal of this study is to obtain a well-characterized collection of translocations for the future development of cytogenetic maps in rye using DNA markers.

\section{Materials and methods}

\section{Plant material and pollen irradiation}

Rye line 1RSL has large blocks of C-heterochromatin in both telomeres of chromosome $1 \mathrm{R}$ (labelled chromosome), while other C-bands are practically absent in the karyotype (Figure 1). Translocations were induced by exposing spikes of this line, near the beginning of anthesis, to $12 \mathrm{~Gy}$ of X-rays. The pollen was then used to pollinate non-irradiated spikes of the same line. The experimental procedure employed (Alvarez et al. 1997)

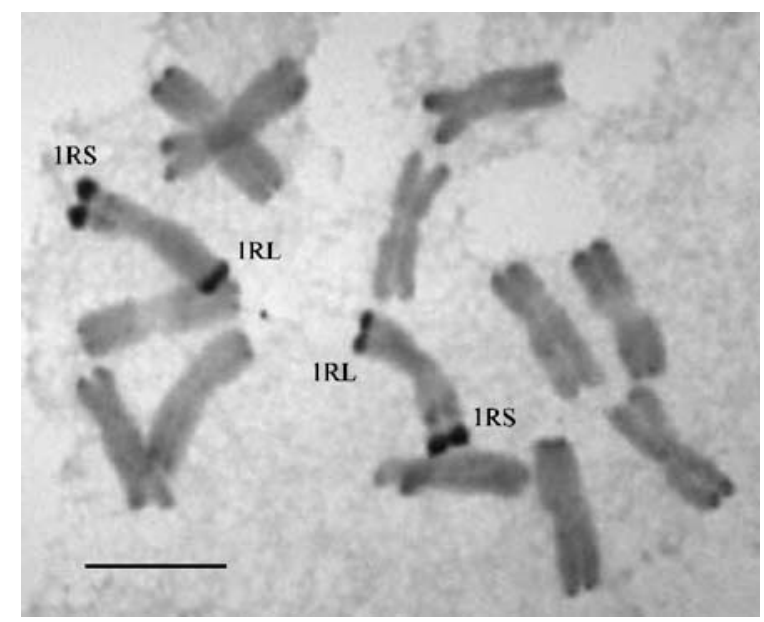

Figure 1. Mitotic metaphase cell of line 1RSL. Chromosome 1R has large $\mathrm{C}$-heterocromatin bands in both telomeres, the other chromosomes being practically free of such C-bands. Bar represents $10 \mu \mathrm{m}$.

is shown in Figure 2. The 20 translocations analysed in this work were those that survived or produced offspring, among 68 plants carrying a putative reciprocal translocation involving chromosome 1R (in eight of them the translocation breakpoint was apparently located in the satellite), identified from the analyses of the C-banded mitotic karyotypes of 1490 seeds obtained from irradiated pollen.

In most of these translocations the non-labelled chromosome involved was identified from the analysis of meiotic configurations of plants proceeding from crosses between the corresponding translocation and the following materials:

1. Plants carrying known translocations (Translocation Tester Set; Wageningen, The Netherlands; Sybenga \& Wolters 1972).

2. Plants carrying large blocks of C-heterochromatin only in the telomeres of some chromosome arms (3RL, 2RS) or a large interstitial C-band in 2RL. All these plant materials with large specific C-bands (as well as line 1RSL) were derived from crosses between lines kindly supplied by Dr A. Lukaszewski (University of California, Riverside).

Plants derived from a ditelocentric line for chromosome 1R (kindly supplied by Dr J. Sybenga, Wageningen, The Netherlands) were used in order to determine the relative position of the centromere of chromosome $1 \mathrm{R}$ in the synaptonemal complexes. 


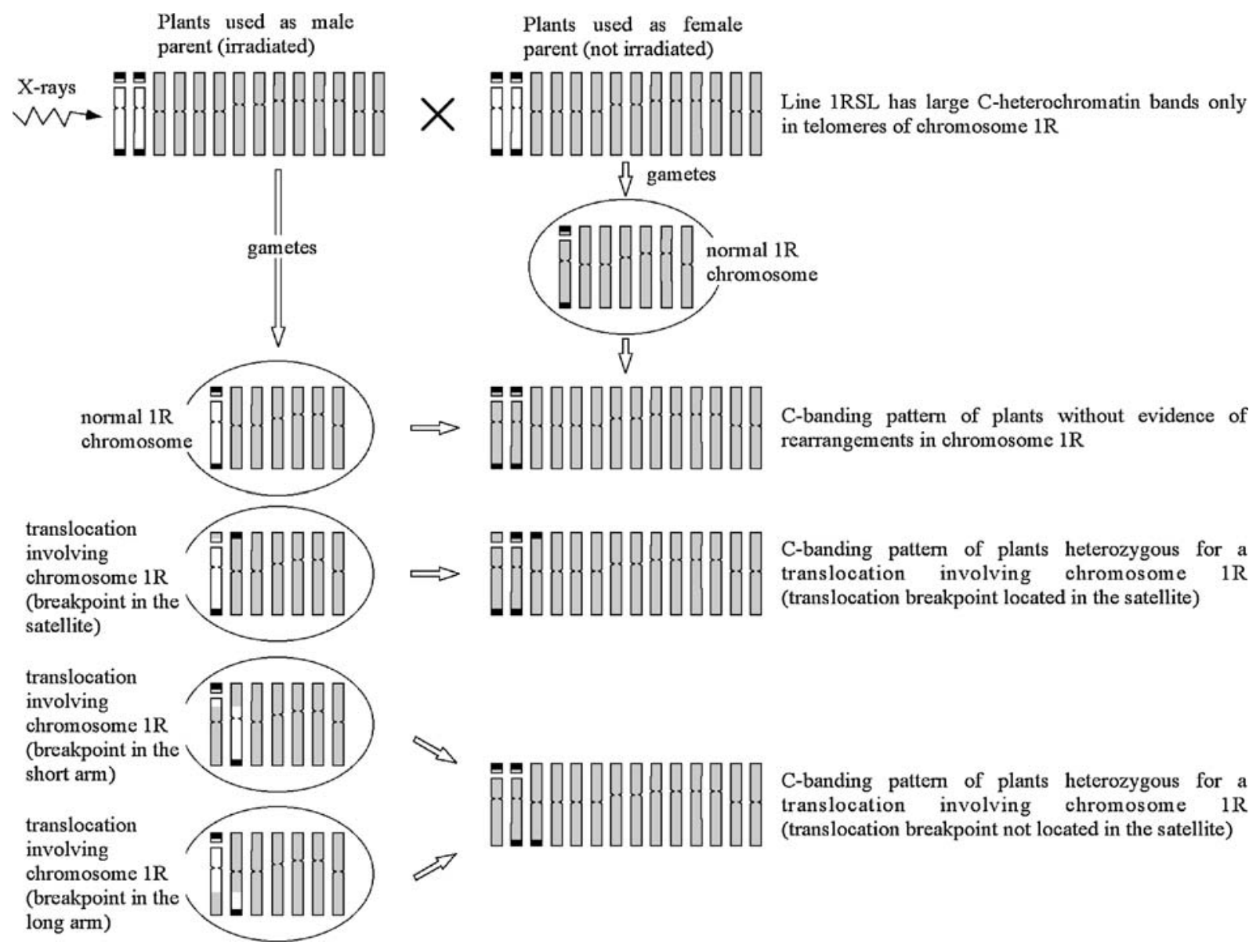

Figure 2. Diagram showing the experimental protocol used. Spikes of line 1RSL were X-irradiated and their pollen was then used to pollinate non-irradiated spikes of the same line. From the relative positions of the large C-heterocromatin bands among the offspring, the presence of a translocation involving the labelled chromosome can be easily detected.

\section{Mitotic and meiotic C-banding analysis}

Root tips of germinated seeds were immersed in tap water at $0^{\circ} \mathrm{C}$ for $24 \mathrm{~h}$ to shorten the chromosomes and fixed in acetic acid-alcohol 1:3 for at least $24 \mathrm{~h}$. The fixed root tips were squashed in acetocarmine, destained in absolute alcohol and C-banded (Giraldez et al. 1979). With acetocarmine squashing, the cell breakage and chromosome dispersion resulting from $45 \%$ acetic acid squashing is avoided, and the quality of the C-banding is sufficiently high to permit the identification of the large $\mathrm{C}$-heterochromatin blocks.

Anthers having PMC at metaphase I were fixed in acetic acid-alcohol 1:3, maintained in the fixative for 1-4 months at $3-4^{\circ} \mathrm{C}$, squashed and C-banded.

\section{Synaptonemal complex analysis}

Synaptonemal complexes of pachytene cells were analysed following the spreading and staining procedures of de Jong et al. (1989) as modified by Naranjo et al. (1989). SC were photographed with the electron microscope (JEOL 2000 EX-II) at $1200 \times$ magnification. The photographs were later digitalized, redrawn and measured using a computer program (Canvas 3.5.2).

\section{Results}

Figure $3 \mathrm{a}-\mathrm{c}$ shows some examples of mitotic cells of translocation heterozygous plants obtained after 


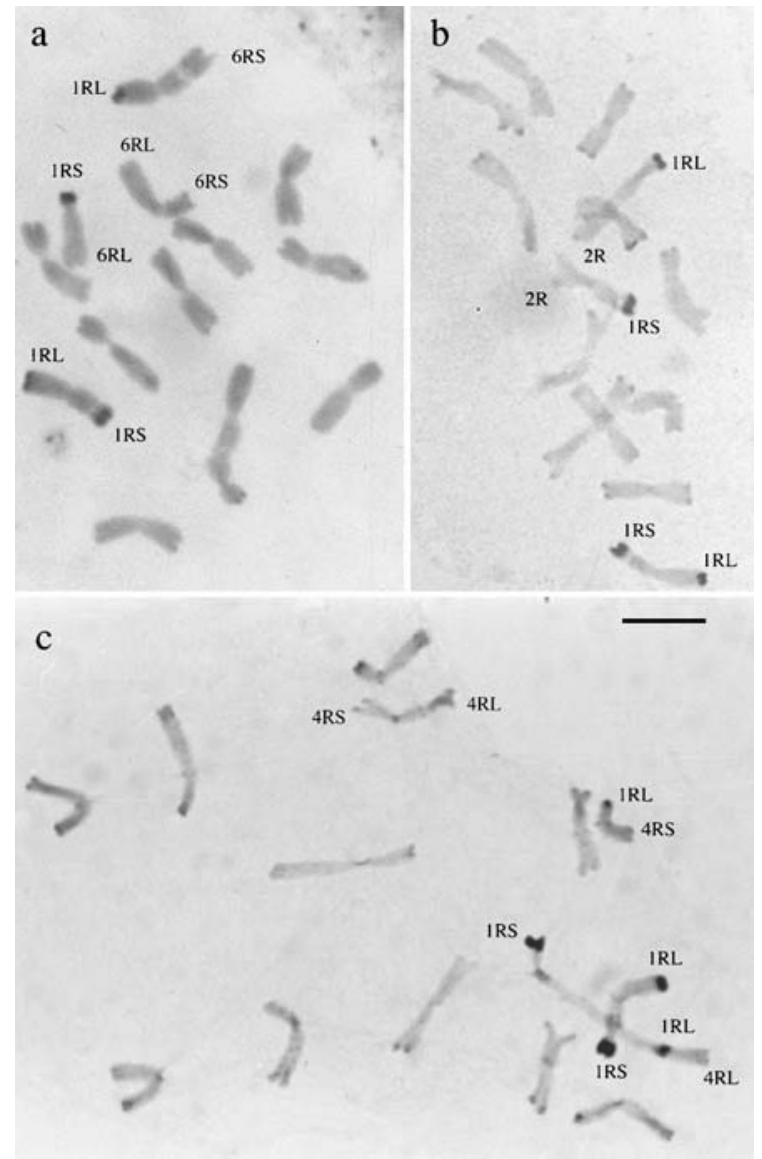

Figure 3. Mitotic metaphase cells of heterozygous plants for reciprocal translocations involving the labelled chromosome $1 \mathrm{R}$. (a) Translocation TX18, with the breakpoints located in the $1 \mathrm{R}$ satellite and in 6RS. (b) Translocation TX35, involving chromosomes 1R and 2R. (c) Translocation TX19, with the breakpoints located in the $1 \mathrm{RL}$ telomere C-heterochromatin and in 4RL. Bar represents $10 \mu \mathrm{m}$.

irradiation of line 1RSL. In these plants the nontranslocated chromosome 1R has two unaltered telomere C-heterochromatin blocks. In most cases translocated chromosomes have only one of such blocks. Translocations having the breakpoint of chromosome $1 \mathrm{R}$ within the satellite can be easily identified by the absence of the telomere C-heterochromatin in this segment (Figure 3a). In translocations in which the breakpoint is located in other chromosome segments, one of the translocated chromosomes carries a telomere C-banded satellite (Figure 3b).

Translocations with the breakpoint within one of the telomere C-bands of chromosome $1 \mathrm{R}$ can be also identified, since in this case one of these C-bands is not complete and is located interstitially in one of the translocated chromosomes (Figure 3c). Among the 20 translocations analysed, three are located in the satellite region and two have their breakpoint within the $1 \mathrm{RL}$ telomere C-band.

The characterization of each translocation included the steps of identifying the other chromosome involved in the translocation and the telomere constitution of the translocated chromosomes and determining the relative position of the translocation breakpoints.

\section{Identification the other chromosome involved in the translocation and telomere constitution of the translocated chromosomes}

Knowledge of the telomere constitutions of the chromosomes involved in a non-complex reciprocal translocation excludes two possibilities concerning the arm location of the translocation breakpoints. For instance, in a translocation involving chromosomes $1 \mathrm{R}$ and $4 \mathrm{R}$, in which the two translocated chromosomes have the telomere constitution 1RS-4RS and 1RL-4RL, the translocation breakpoint can be located either in the arms 1RS and 4RL, or in the arms 1RL and $4 \mathrm{RS}$, the other two possibilities, 1RS and 4RS or $1 R L$ and 4RL, being excluded.

In translocations involving chromosomes $4 \mathrm{R}, 5 \mathrm{R}$ or $6 \mathrm{R}$, the identification of the telomere constitution of translocated chromosomes was possible in all cases after the analysis of C-banded mitotic metaphases, since chromosomes $4 \mathrm{R}, 5 \mathrm{R}$ and $6 \mathrm{R}$ have specific C-banding patterns and significant size differences between their chromosome arms. In translocations involving chromosomes $2 \mathrm{R}, 3 \mathrm{R}$ or $7 \mathrm{R}$, mitotic identification was questionable due to the high similarity of C-banding pattern and arm ratio of these three chromosomes. These translocations were crossed with lines carrying either specific C-bands (located in chromosome arms 3RL or 2RL) or previously characterized translocations. The chromosome involved in the translocation was identified in all cases after analysis of the meiotic configurations formed in the progeny of such crosses, but the identification of the telomere constitution of the translocated chromosomes was possible only in one case (translocation TX17). 


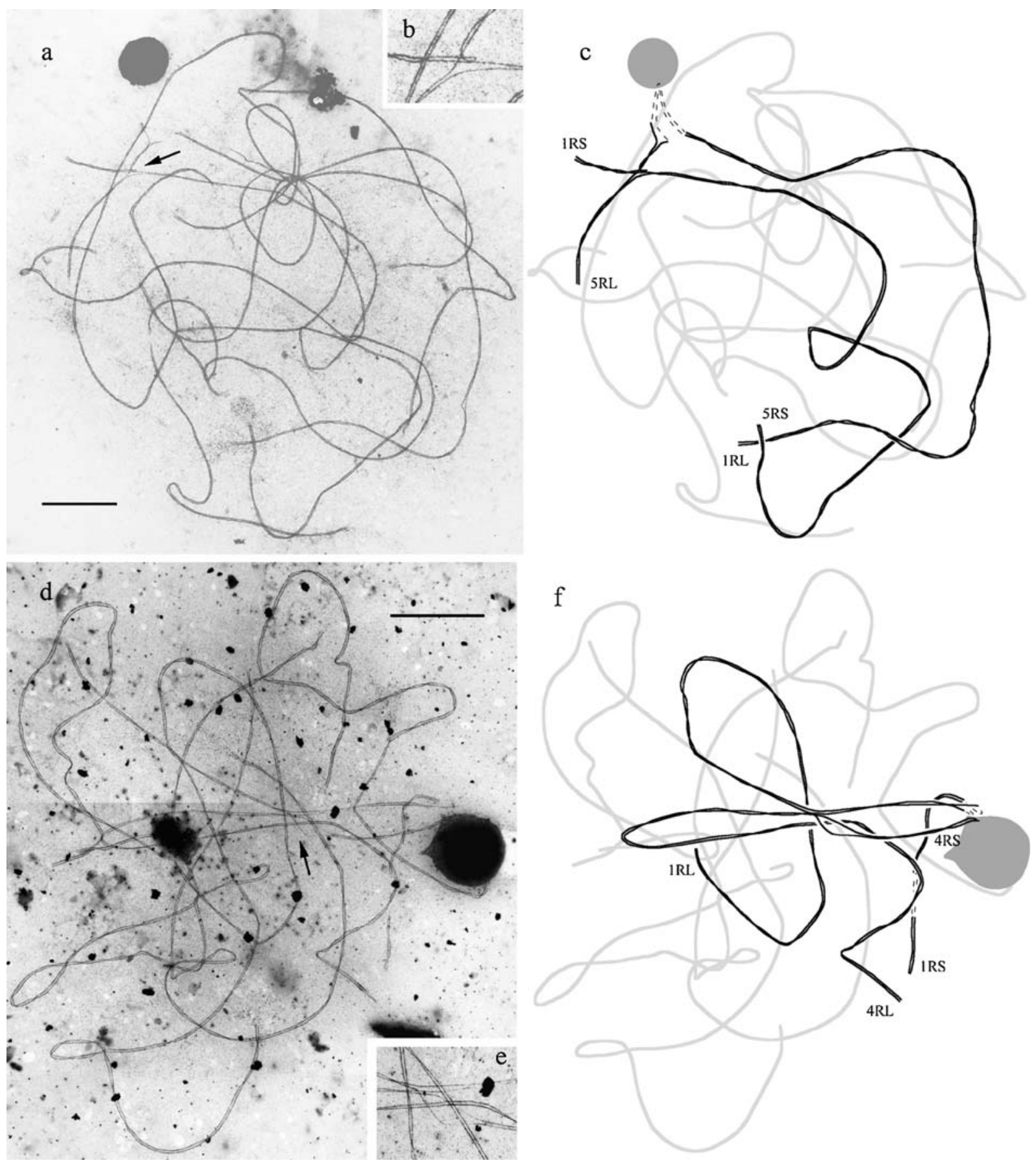

Figure 4. (a) Electron micrograph of the spread synaptonemal complexes of a pachytene nucleus of a plant heterozygous for translocation TX04, with the breakpoints located in the $1 \mathrm{R}$ satellite and in 5RL. The arrow shows the partner exchange (translocation breakpoint) detailed in (b) and the corresponding quadrivalent (black) is interpreted in (c). (d) Electron micrograph of the spread synaptonemal complexes of a pachytene nucleus of a plant heterozygous for translocation TX10, with the breakpoints located in the 1RS and in 4RL. The arrow shows the partner exchange (translocation breakpoint) detailed in (e) and the corresponding quadrivalent (black) is interpreted in (f). Bars represent $10 \mu \mathrm{m}$. 
Determination of the physical position

of the translocation breakpoints

Figure 4 shows the spread of SC from pachytene nuclei of a translocation heterozygote with a breakpoint in the satellite of chromosome 1R (TX04) and a translocation heterozygote with a breakpoint located in a different $1 \mathrm{R}$ chromosome region (TX06). The relative positions of the NOR and the translocation breakpoint (TB) delimit five segments in the pachytene quadrivalents formed in translocation heterozygotes involving chromosome $1 \mathrm{R}$. This is illustrated in Figure 5. In translocations having the breakpoint in the satellite (Figure 5a), the chromosome 1R segments, TB-NOR and NOR-1RL telomere, as well as the segment carrying the centromere of the other chromosome involved in the translocation, can be unequivocally identified (segments Ib, Ic and IIb, respectively, in Figure 5a). The remaining two segments of the pachytene quadrivalent corresponding to the chromosome 1R segment, TB-1RS telomere and to the acentric translocated segment of the other chromosome involved (segments Ia and IIa, respectively, in Figure 5a), were identified based on their relative
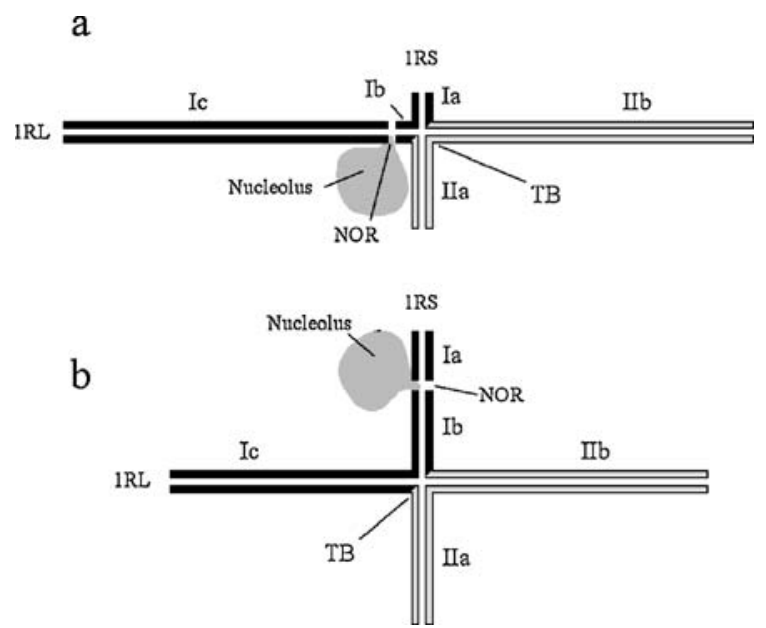

Figure 5. Schematic representation of the two possible SC quadrivalents observed in $1 \mathrm{R}$ translocations, indicating the segments that can be distinguished. The normal chromosome $1 \mathrm{R}$ is formed by segments $\mathrm{Ia}+\mathrm{Ib}+\mathrm{Ic}$. The other chromosome involved in the translocation is formed by segments IIa + IIb. (a) Quadrivalent formed by translocations with the $1 \mathrm{R}$ breakpoint (TB) located in the satellite. The translocated chromosomes are constituted by segments Ia + IIb and IIa + Ib + Ic, respectively. (b) Quadrivalent formed by translocations with the $1 \mathrm{R}$ breakpoint (TB) not located in the satellite. The translocated chromosomes are constituted by segments Ia + Ib + IIb and Ic + IIa, respectively. lengths. In translocations in which the breakpoint is not located in the satellite (Figure $5 \mathrm{~b}$ ), the $1 \mathrm{R}$ chromosome segments, 1RS telomere-NOR (corresponding to the satellite) and NOR-TB, as well as the opposite segment corresponding to the other chromosome involved in the translocation (segments $\mathrm{Ia}, \mathrm{Ib}$ and IIa, respectively, in Figure 5b), can also be identified unambiguously. The assignment of the other two quadrivalent segments (Ic and IIb in Figure 5b) to chromosome 1R (segment TB-1RL telomere) and to a part of the other chromosome involved in the translocation, respectively, was based on their relative lengths. Since all rye chromosomes are not very different in length, chromosome $1 \mathrm{R}$ being the shortest of the complement (Alvarez et al. 1994), the length of $\mathrm{Ia}+\mathrm{Ib}+\mathrm{Ic}$ (corresponding to chromosome 1R) should be smaller but not very different from that of IIa + IIb (corresponding to the other chromosome involved in the translocation).

Table 1 shows the relative length of the SC segments of the quadrivalents formed in pachytene cells of heterozygotes for the 20 translocations analysed. A physical map including the relative positions of the corresponding 40 translocation breakpoints is shown in Figure 6. The technique of SC spreading in rye does not allow the identification of the centromere. In order to determine the relative position of $1 \mathrm{R}$ centromere in the $\mathrm{SC}$, pachytene cells of plants ditelocentric for chromosome 1R (having one of the 1R chromosomes replaced by the corresponding telocentric chromosomes $1 \mathrm{RS}$ and $1 \mathrm{RL}$ ) were analysed. In some of these cells (Figure 7) the two telocentrics and the normal chromosome $1 \mathrm{R}$ were slightly unpaired at the centromere region and the relative position of this region was determined. The results indicate that the segment NOR-centromere corresponds to $25.3 \pm 1.6 \%$ of the chromosome. This value agrees with that obtained by Qi et al. (1988) and Alonso-Blanco et al. (1993a). The relative positions of centromeres in chromosomes 2R-7R, shown in Figure 6, were taken from Alvarez et al. (1994). The SC segments carrying the centromeres of the two chromosomes involved in each translocation are also indicated in Table 1.

In translocation heterozygotes in which the $1 \mathrm{R}$ breakpoint is not located in the satellite, the $1 \mathrm{R}$ chromosome arm location of the translocation breakpoint can also be analysed in mitotic metaphases, since segments NOR-centromere and centromere-1RL 
Table 1. Relative length (\% $\pm \mathrm{SE}$ ) of the synaptonemal complex (SC) segments of the quadrivalents formed in pachytene cells of heterozygotes for the different translocations involving chromosome 1R. For each translocation, segments Ia $+\mathrm{Ib}+\mathrm{Ic}$ correspond to the SC formed by the normal chromosome $1 \mathrm{R}$ and segments IIa + IIb correspond to the SC formed by the other chromosome involved in the translocation (see Figure 5)

\begin{tabular}{|c|c|c|c|c|c|c|c|c|}
\hline \multirow[b]{2}{*}{ Translocation } & \multirow{2}{*}{$\begin{array}{l}\text { Other } \\
\text { chromosome } \\
\text { involved in the } \\
\text { translocation }^{c}\end{array}$} & \multicolumn{5}{|c|}{ Quadrivalent segments } & \multirow{2}{*}{$\begin{array}{l}\text { Relative SC mean } \\
\text { length }(\%) \text { of the } \\
\text { chromosomes } \\
\text { forming bivalents }\end{array}$} & \multirow{2}{*}{$\begin{array}{l}\text { Number of } \\
\text { pachytene } \\
\text { cells }\end{array}$} \\
\hline & & Ia & $\mathrm{Ib}$ & Ic & IIa & $\mathrm{IIb}$ & & \\
\hline $\mathrm{TX}_{01}{ }^{\mathrm{b}}$ & $3 R$ & $1.97 \pm 0.07$ & $2.64 \pm 0.51$ & $8.81 \pm 0.63$ & $5.40 \pm 0.54$ & $10.01 \pm 0.60$ & $14.23 \pm 0.16$ & 7 \\
\hline $\mathrm{TX} 02^{\mathrm{b}}$ & $7 \mathrm{R}$ & $1.99 \pm 0.06$ & $11.57 \pm 0.74$ & $0.05 \pm 0.03$ & $11.35 \pm 0.25$ & $0.19 \pm 0.04$ & $14.97 \pm 0.22$ & 6 \\
\hline $\mathrm{TX}_{0} 3^{\mathrm{b}}$ & $2 \mathrm{R}$ & $2.28 \pm 0.13$ & $2.94 \pm 0.41$ & $7.84 \pm 0.42$ & $6.37 \pm 0.48$ & $8.65 \pm 0.37$ & $14.38 \pm 0.14$ & 12 \\
\hline $\mathrm{TX}_{0} 4^{\mathrm{a}}$ & 5R(1RL-5RL) & $1.72 \pm 0.18$ & $0.37 \pm 0.17$ & $11.66 \pm 0.36$ & $2.59 \pm 0.16$ & $12.89 \pm 0.26$ & $14.15 \pm 0.14$ & 9 \\
\hline $\mathrm{TX}_{05^{\mathrm{b}}}$ & $3 \mathrm{R}$ & $1.98 \pm 0.43$ & $3.13 \pm 0.41$ & $9.03 \pm 0.60$ & $5.10 \pm 0.47$ & $9.10 \pm 0.33$ & $14.33 \pm 0.20$ & 4 \\
\hline TX06 $^{\mathrm{b}}$ & 4R(1RS-4RL) & $2.33 \pm 0.16$ & $3.73 \pm 1.07$ & $7.36 \pm 1.40$ & $5.92 \pm 1.29$ & $8.80 \pm 1.07$ & $14.37 \pm 0.15$ & 5 \\
\hline TX08 ${ }^{\mathrm{b}}$ & $2 \mathrm{R}$ & $1.89 \pm 0.07$ & $2.53 \pm 0.38$ & $9.19 \pm 0.52$ & $6.43 \pm 0.58$ & $8.30 \pm 0.43$ & $14.33 \pm 0.11$ & 14 \\
\hline TX09 & 5R(1RS-5RL) & $1.85 \pm 0.08$ & $2.53 \pm 0.47$ & $8.57 \pm 0.70$ & $4.88 \pm 0.85$ & $10.90 \pm 0.91$ & $14.25 \pm 0.22$ & 9 \\
\hline $\mathrm{TX} 10^{\mathrm{b}}$ & $4 \mathrm{R}(1 \mathrm{RS}-4 \mathrm{RS})$ & $2.16 \pm 0.14$ & $2.51 \pm 0.72$ & $9.83 \pm 0.72$ & $3.60 \pm 0.46$ & $10.77 \pm 0.56$ & $14.23 \pm 0.24$ & 4 \\
\hline $\mathrm{TX} 12^{\mathrm{b}}$ & $6 \mathrm{R}(1 \mathrm{RS}-6 \mathrm{RL})$ & $1.46 \pm 0.21$ & $1.63 \pm 0.27$ & $8.79 \pm 0.86$ & $5.25 \pm 0.73$ & $10.59 \pm 0.69$ & $14.46 \pm 0.22$ & 5 \\
\hline $\mathrm{TX} 15^{\mathrm{b}}$ & $7 \mathrm{R}$ & $2.32 \pm 0.45$ & $2.91 \pm 1.21$ & $9.42 \pm 2.16$ & $6.91 \pm 1.30$ & $6.98 \pm 1.67$ & $14.29 \pm 0.23$ & 3 \\
\hline TX $16^{\mathrm{b}}$ & $7 \mathrm{R}$ & $2.60 \pm 0.34$ & $0.78 \pm 0.30$ & $9.97 \pm 0.14$ & $4.76 \pm 0.36$ & $8.48 \pm 0.46$ & $14.68 \pm 0.23$ & 3 \\
\hline $\mathrm{TX} 17^{\mathrm{b}}$ & 2R(1RS-2RS) & $1.87 \pm 0.23$ & $2.45 \pm 0.42$ & $8.65 \pm 0.69$ & $5.72 \pm 0.61$ & $10.09 \pm 0.69$ & $14.24 \pm 0.22$ & 5 \\
\hline TX $18^{\mathrm{a}}$ & $6 \mathrm{R}(1 \mathrm{RL}-6 \mathrm{RS})$ & $0.97 \pm 0.49$ & $1.96 \pm 0.24$ & $11.82 \pm 0.71$ & $2.25 \pm 0.40$ & $11.79 \pm 0.63$ & $14.24 \pm 0.20$ & 7 \\
\hline TX19 & 4R(1RS-4RL) & $2.16 \pm 0.12$ & $11.36 \pm 0.31$ & $0.84 \pm 0.19$ & $11.19 \pm 0.55$ & $4.38 \pm 0.45$ & $14.01 \pm 0.13$ & 9 \\
\hline $\mathrm{TX} 26^{\mathrm{b}}$ & $3 \mathrm{R}$ & $2.17 \pm 0.32$ & $3.19 \pm 0.53$ & $9.37 \pm 0.51$ & $6.26 \pm 0.95$ & $7.11 \pm 0.37$ & $14.38 \pm 0.30$ & 5 \\
\hline $\mathrm{TX} 31^{\mathrm{b}}$ & $2 \mathrm{R}$ & $1.82 \pm 0.09$ & $3.58 \pm 0.48$ & $7.81 \pm 0.42$ & $4.53 \pm 0.64$ & $9.74 \pm 0.39$ & $14.50 \pm 0.22$ & 11 \\
\hline $\mathrm{TX} 34^{\mathrm{b}}$ & 6R(1RS-6RL) & $1.70 \pm 0.24$ & $5.25 \pm 0.72$ & $7.07 \pm 0.91$ & $7.44 \pm 1.14$ & $8.70 \pm 1.16$ & $13.93 \pm 0.21$ & 5 \\
\hline $\mathrm{TX} 35^{\mathrm{b}}$ & $2 \mathrm{R}$ & $2.17 \pm 0.13$ & $2.19 \pm 0.34$ & $9.74 \pm 0.40$ & $6.25 \pm 0.70$ & $8.59 \pm 0.52$ & $14.21 \pm 0.30$ & 6 \\
\hline $\mathrm{TX}^{\mathrm{a}} 6^{\mathrm{a}}$ & 6R(1RL-6RL) & $0.69 \pm 0.22$ & $1.18 \pm 0.62$ & $11.23 \pm 0.63$ & $1.73 \pm 0.33$ & $12.98 \pm 1.04$ & $14.44 \pm 0.34$ & 3 \\
\hline
\end{tabular}

${ }^{\mathrm{a}}$ Translocations with the $1 \mathrm{R}$ chromosome breakpoint located in the satellite.

${ }^{\mathrm{b}}$ Translocations with the $1 \mathrm{R}$ chromosome breakpoint not located in the satellite.

${ }^{\mathrm{c}}$ Telomere constitution of the translocated chromosome carrying the satellite is indicated between brackets.

telomere can be clearly distinguished in the normal $1 \mathrm{R}$ chromosome and in the corresponding translocated chromosomes (Figure 3). Table 2 shows the comparison between the relative lengths of these segments in eight translocation heterozygotes in which at least four complete and not distorted mitotic metaphase cells could be obtained. In four cases (TX10, TX16, TX17 and TX31), significant differences for the relative length of segment NOR-centromere were found between the normal and the translocated chromosomes. This indicates that the corresponding $1 \mathrm{R}$ breakpoints are located in the short arm, and that the lengths of the segments between the breakpoint and the centromere are significantly different in the two chromosomes involved in each translocation. In the remaining four cases (TX08, TX15, TX34 and TX35), differences were not significant, indicating that the segments between the breakpoint and the centromere are similar in both chromosomes involved in each translocation.

\section{Discussion}

Among the 20 translocations involving chromosome $1 \mathrm{R}$ characterized in this work, the number of cases in which each non-labelled chromosome is involved ranges from two to five (Figure 6). This is in agreement with the random assumption of chromosomes $2 \mathrm{R}$ to $7 \mathrm{R}$ having the same probability $(1 / 6)$ of being involved in a translocation with chromosome $1 \mathrm{R}$. However, the within-chromosome positions of the translocation breakpoints are not randomly distributed. This is particularly evident in chromosome $1 \mathrm{R}$ (Figure 6), in which three breakpoints are located in the satellite, 13 in the segment NOR-centromere (all except one located in the proximal half of this segment), and only four in the long arm, two of them located near the centromere and the other two located near the telomere. Of course, a between-cell variation in the relative position of the translocation breakpoint within the quadrivalent was observed (see standard 


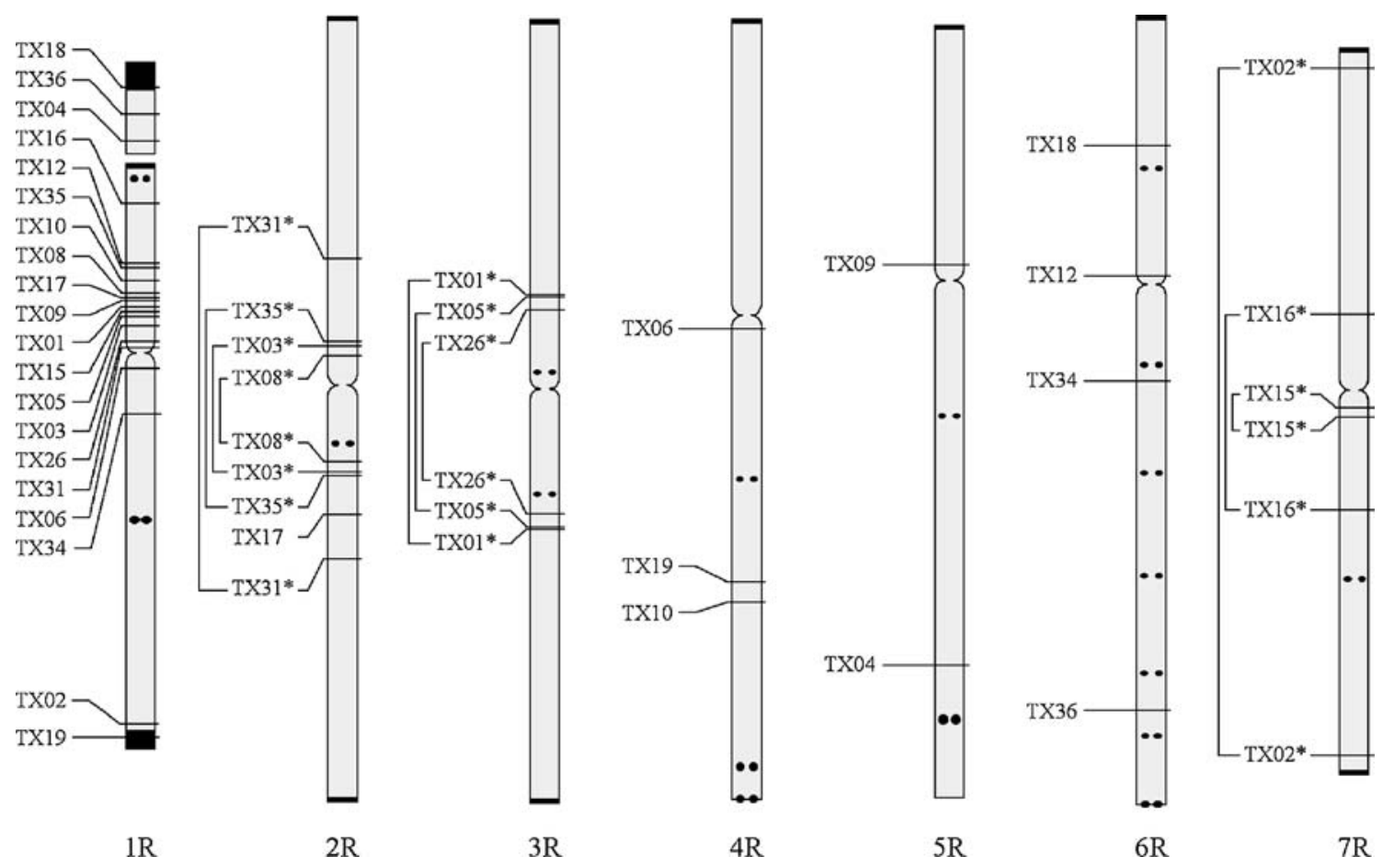

Figure 6. Physical map (relative positions) of the breakpoints of the 20 translocations analysed. The arm locations of the translocation breakpoints indicated by an asterisk were not unequivocally determined. The two alternative positions of these breakpoints are indicated.
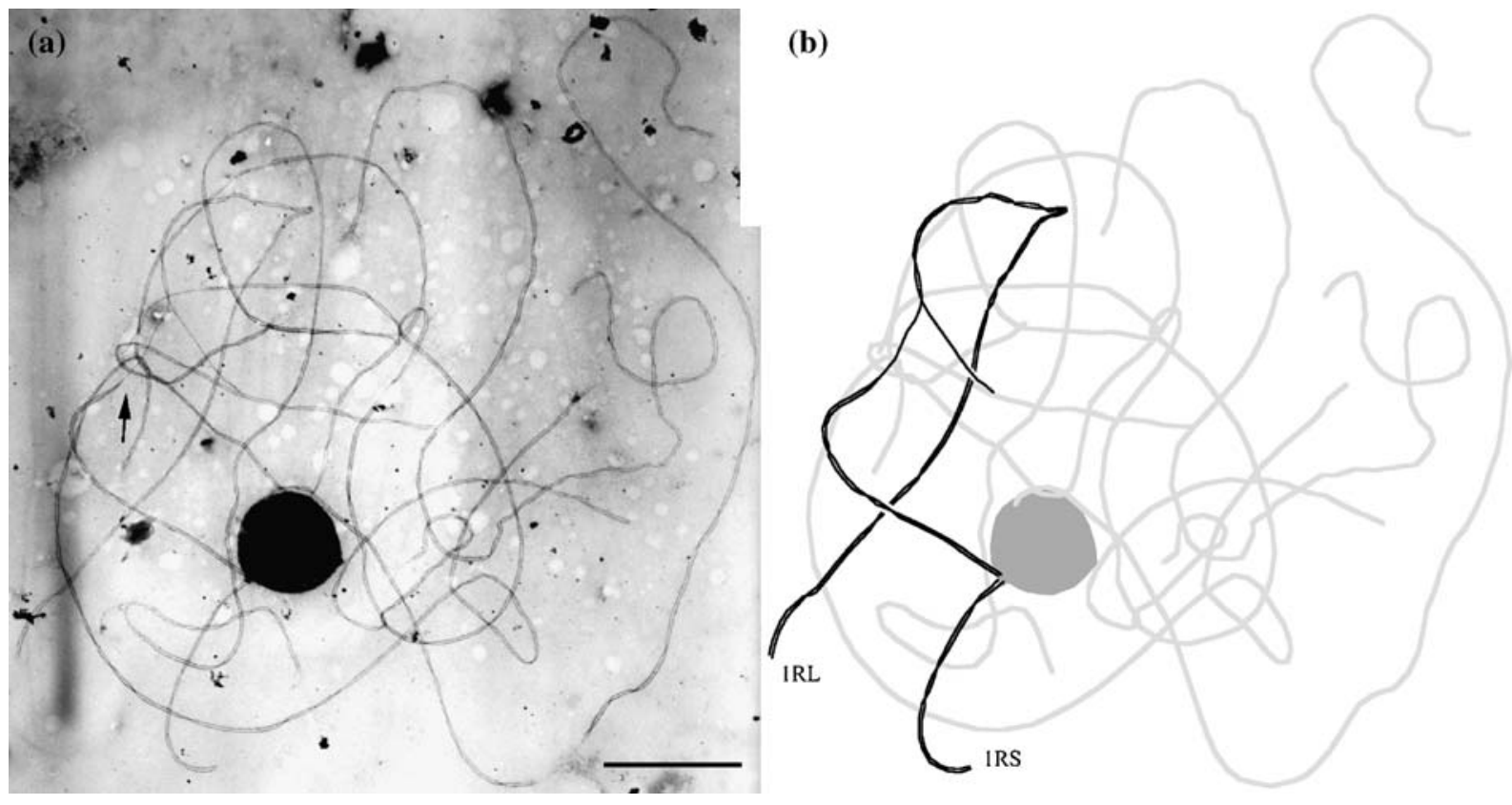

Figure 7. Pachytene nucleus of a plant ditelocentric for chromosome $1 \mathrm{R}$ (having one of the $1 \mathrm{R}$ chromosomes replaced by the corresponding telocentric chromosomes 1RS and 1RL). (a) Electron micrograph of spread synaptonemal complexes. (b) Interpretation of the trivalent formed by the $1 \mathrm{R}, 1 \mathrm{RS}$ and $1 \mathrm{RL}$ chromosomes. The telocentric chromosome $1 \mathrm{RL}$ and the normal chromosome $1 \mathrm{R}$ are unpaired at the centromere region (arrow). Bar represents $10 \mu \mathrm{m}$. 
Table 2. The comparison between the relative mitotic metaphase lengths (\%) of segments NOR-centromere and centromere-1RL telomere in the normal chromosome $1 \mathrm{R}$ and in the corresponding translocated chromosomes of eight translocation heterozygotes

\begin{tabular}{|c|c|c|c|c|c|c|c|c|c|}
\hline \multirow[b]{2}{*}{ Translocation } & \multirow[b]{2}{*}{$\begin{array}{l}\text { Number } \\
\text { of cells }\end{array}$} & \multicolumn{4}{|c|}{ Relative length of segment NOR-centromere } & \multicolumn{4}{|c|}{ Relative length of segment centromere-1RL telomere } \\
\hline & & $\begin{array}{l}\text { Normal } \\
\text { chromosome } \\
1 \mathrm{R}\end{array}$ & $\begin{array}{l}\text { Translocated } \\
\text { chromosome }\end{array}$ & $\begin{array}{l}\text { Paired } \\
t \text {-test }\end{array}$ & & $\begin{array}{l}\text { Normal } \\
\text { chromosome } \\
1 \mathrm{R}\end{array}$ & $\begin{array}{l}\text { Translocated } \\
\text { chromosome }\end{array}$ & $\begin{array}{l}\text { Paired } \\
t \text {-test }\end{array}$ & \\
\hline TX08 & 10 & 2.06 & 2.33 & 1.59 & n.s. & 3.90 & 4.17 & 1.75 & n.s. \\
\hline TX10 & 7 & 2.47 & 1.69 & 3.83 & $* *$ & 3.88 & 3.51 & 1.31 & n.s. \\
\hline TX15 & 8 & 2.00 & 2.41 & 1.98 & n.s. & 4.05 & 4.14 & 0.65 & n.s. \\
\hline TX16 & 8 & 2.54 & 1.89 & 3.00 & $* *$ & 4.10 & 4.31 & 1.12 & n.s. \\
\hline TX17 & 7 & 2.36 & 3.37 & 3.70 & $* *$ & 4.04 & 4.14 & 0.26 & n.s. \\
\hline TX31 & 6 & 2.16 & 1.72 & 8.05 & $* * *$ & 3.76 & 3.86 & 1.32 & n.s. \\
\hline TX34 & 10 & 1.98 & 2.36 & 1.40 & n.s. & 4.41 & 4.16 & 0.98 & n.s. \\
\hline TX35 & 4 & 1.82 & 1.89 & 0.52 & n.s. & 3.99 & 4.22 & 0.33 & n.s. \\
\hline
\end{tabular}

$* * *, p<0.001 ; * *, 0.01>p>0.001 ;$ n.s., non- significant $(p>0.05)$.

errors in Table 1), and the actual positions of the breakpoints can be somewhat different from those shown in Figure 6. However, the variation found cannot explain the absence of breakpoints in most of the $1 \mathrm{R}$ long arm.

In order to explain this situation the possibility of big differences in the relative length of specific $1 \mathrm{R}$ SC segments, when compared to those of the $1 \mathrm{R}$ mitotic chromosome, could be considered. However, there are several reasons to exclude this possibility. In serial reconstruction of early rye meiotic nuclei, Abirached-Darmency et al. (1983) observed a good correlation between the SC arm ratio of pachytene bivalents and the light microscopic data of meiotic

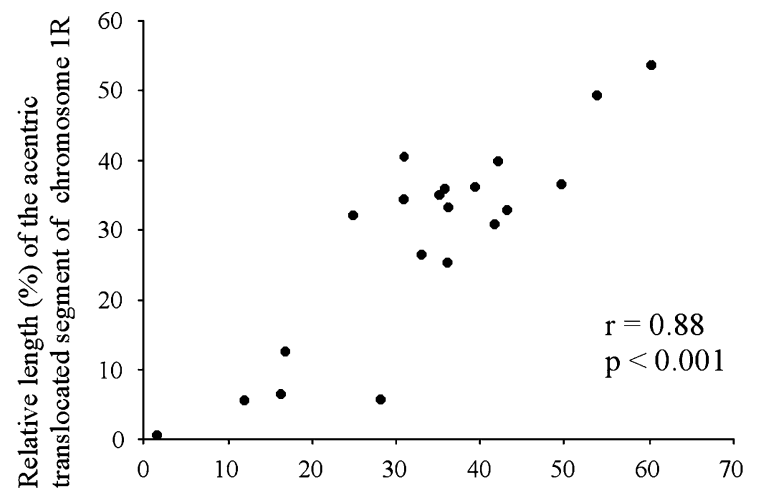

Relative length (\%) of the acentric translocated segment of the other chromosome involved in the translocation

Figure 8 . The relative length of the $1 \mathrm{R}$ acentric translocated segment of each translocation, plotted against the relative length of the corresponding acentric translocated segment of the other chromosome involved. and mitotic chromosomes. Also, in agreement with previous reports (Qi et al. 1988, Alonso-Blanco et al. 1993a) the SC analyses of ditelocentric heterozygotes made in this work show a good correspondence between the arm ratios of the pachytene bivalents and those of the corresponding mitotic chromosomes. Finally, a good agreement between the results obtained from mitotic measurements (Table 2) and those of SC analyses (Figure 6) was obtained. Both approaches locate translocations TX10, TX16, TX17 and TX31 in the short arm, and indicate that the lengths of the segments between the breakpoint and the centromere are significantly different in the two chromosomes involved in each translocation. Also, the results obtained under the two approaches for translocations TX08, TX15, TX34 and TX35, indicate that the segments between the breakpoint and the centromere are similar in both chromosomes involved in each translocation.

As indicated before, the 20 translocations analysed in this work were those that survived or produced offspring, among 68 plants carrying a putative reciprocal translocation involving chromosome $1 \mathrm{R}$, obtained from irradiation experiments. The nonrandom distribution of such breakpoints could be the result of a selection, if the location of the $1 \mathrm{R}$ translocation breakpoints is related with viability. A higher viability of translocations having the breakpoint located near the centromere, in the telomere $\mathrm{C}$-heterochromatin blocks, or in other regions having a lower gene density, could be a realistic possibility.

The independence between the relative locations of the two breakpoints (in $1 \mathrm{R}$ and in the other chromo- 
some involved, respectively) of each translocation, can also be tested. Figure 8 shows the relative length of the $1 \mathrm{R}$ acentric translocated segment of each translocation, plotted against the relative length of the corresponding acentric translocated segment of the other chromosome involved (see also Table 1). The high positive correlation found $(r=0.88 ; p<0.001)$ indicates the existence of a strong tendency for the two breakpoints of a translocation towards being located at a similar distance from the telomeres of the arms in which they are located, giving rise to translocated chromosomes having lengths and arm ratios similar to those of the normal ones. This is in agreement with the results of Gecheff (1996) in barley, in which a large portion of the gamma-induced translocations resulted in the exchange of approximately equal chromosome segments. If a close proximity between the two breaks originating a reciprocal translocation is required, the tendency observed could be the result of a non-random spatial arrangement of chromosomes in the interphase nucleus. A remnant of telophase arrangement, with the telomeres of all chromosomes located at or near a restricted part of the nuclear envelope, and the chromosome arms remaining parallel to each other (Rabl orientation), could explain the observed tendency.

Translocations can be an excellent tool for developing cytogenetic maps. In barley, a cytologically integrated physical map was developed by Künzel et al. (2000) using microdissected translocation chromosomes for PCR with sequence-tagged site primers derived from more than 300 genetically mapped RFLP probes. Since microdissection techniques require the identification of the unstained translocated chromosomes, only translocations showing size differences affecting such chromosomes can be used. The advantage of rye translocations involving chromosome $1 \mathrm{R}$ is that chromosomes carrying the satellite can be unequivocally identified in all cases.

\section{Acknowledgements}

Susana Catarino held a fellowship (PRAXIS XXI/ BD/21344/99) from the Fundação para a Ciência e Tecnologia (FCT), Portugal. The authors thank Dr Reinerio Rodríguez, Servicio de Oncología Radioterapica, Hospital General de Asturias, for the facilities provided, and Carlos Alvarez for excellent technical assistance.

\section{References}

Abirached-Darmency M, Zickler D, Cauderon Y (1983) Synaptonemal complex and recombination nodules in rye (Secale cereale). Chromosoma 88: 299-306.

Alonso-Blanco C, Goicoechea PG, Roca A, Giraldez R (1993a) A cytogenetic map on the entire length of rye chromosome $1 \mathrm{R}$, including one translocation breakpoint, three isozyme loci and four C-bands. Theor Appl Genet 85: 735-744.

Alonso-Blanco C, Goicoechea PG, Roca A, Giraldez R (1993b) Genetic linkage between cytological markers and the seed storage protein loci $\mathrm{Sec} 2$ [Gli-R2] and $\mathrm{Sec} 3$ [Glu-Rl] in rye. Theor Appl Genet 87: 321-327.

Alonso-Blanco C, Goicoechea PG, Roca A, Alvarez E, Giraldez R (1994) Genetic mapping of cytological and isozyme markers on chromosomes 1R, 3R, 4R and 6R of rye. Theor Appl Genet $\mathbf{8 8}$ : 208-214.

Alvarez E, Alonso-Blanco C, Roca A, Goicoechea PG, Giraldez R (1994) Physical mapping of translocation breakpoints in rye by means of synaptonemal complex analysis. Theor Appl Genet $\mathbf{8 9}$ : 33-41.

Alvarez E, Alonso-Blanco C, Suárez RG, Ferreira JJ, Roca A, Giraldez R (1997) A strategy for detecting chromosome-specific rearrangements in rye. Genome 40: 451-457.

de Jong JH, van Eden J, Sybenga J (1989) Synaptonemal complex formation and metaphase I configuration patterns in a translocation heterozygote of rye (Secale cereale). Genome 32: 72-81.

de Vries JN, Sybenga J (1984) Chromosomal location of 17 monogenically inherited morphological markers in rye (Secale cereale L.) using the translocation tester set. $Z$ Pflanzenzuecht 92: $117-139$.

Gecheff KI (1996) Production and identification of new structural chromosome mutations in barley (Hordeum vulgare L.). Theor Appl Genet 92: 777-781.

Giraldez R, Cermeño MC, Orellana J (1979) Comparison of $\mathrm{C}$-banding pattern in the chromosomes of inbred lines and open pollinated varieties of rye. Z. Pflanzenzüchtg 83: 40-48.

Hoisington DA, Coe EH (1990) Mapping in maize using RFLPs. In Gustafson JP, ed., Gene Manipulation in Plant Improvement, vol. II. New York: Plenum Press, pp. 331-352.

Künzel G (1992) Coordinator's report: translocations and balanced tertiary trisomics. Barley Genet Newslett 22: 80-102.

Künzel G, Korzun L, Meister A (2000) Cytologically integrated physical restriction fragment length polymorphism maps for barley genome based on translocation breakpoints. Genetics 154: 397-412.

Linde-Laursen I (1988) Giemsa C-banding of barley chromosomes. Localization of breakpoints in 70 reciprocal translocations. Hereditas 108: 65-76.

Longley AE (1961) Breakage points for four translocations series and other corn chromosome aberrations maintained at the California Institute of Technology. U.S. Dep. Agric. Res. Serv. Rep. No. 34-16: 1-40.

Naranjo T, Roca A, Goicoechea PG, de Jong JH, Smilde WD (1989) Comparison between synaptonemal complexes at pachytene and chromosome association at metaphase I in heterozygotes for a 'non-reciprocal' translocation of rye. Genome 32: 983-991.

Qi LL, Sybenga J, de Jong JH (1988) Synaptonemal complex pairing and metaphase I association in a telo-substituted 
telotrisomic of rye (Secale cereale L.). Chromosoma 97: 88-95.

Ramulu KS, Sybenga J (1985) Genetic background damage accompanying reciprocal translocations induced by X-rays and fission neutrons in Arabidopsis and Secale. Mutation Res 149: 421-430.

Sorokin A, Marthe F, Houben A, Pich U, Graner A, Künzel G (1994) Polymerase chain reaction mediated localization of RFLP clones to microisolated translocation chromosomes of barley. Genome 37: 550-555.
Sybenga J (1995) Aneuploid and other cytological tester sets in rye. Euphytica 83: 53-61.

Sybenga J, Wolters AHG (1972) The classification of the chromosomes of rye (Secale cereale L.): a translocation tester set. Genetica 43: 453-464.

Sybenga J, Parmar S, van Eden J, Shewry P (1990) Mapping seed storage protein loci $\mathrm{Sec}-1$ and $\mathrm{Sec}-3$ in relation to five chromosomal rearrangements in rye (Secale cereale L.). Theor Appl Genet 79: 201-208. 